\title{
The Impact That Number of Analyzed Metastatic Breast Cancer Lesions Has on Response Assessment by ${ }^{18}$ F-FDG PET/CT Using PERCIST
}

\author{
Katja Pinker ${ }^{1,2}$, Christopher C. Riedl ${ }^{1}$, Leonard Ong ${ }^{1}$, Maxine Jochelson ${ }^{3}$, Gary A. Ulaner ${ }^{1}$, Heather McArthur ${ }^{4}$, \\ Maura Dickler ${ }^{5}$, Mithad Gönen ${ }^{6}$, and Wolfgang A. Weber ${ }^{1}$ \\ ${ }^{I}$ Molecular Imaging and Therapy Service, Department of Radiology, Memorial Sloan Kettering Cancer Center, New York, \\ New York; ${ }^{2}$ Division of Molecular and Gender Imaging, Department of Biomedical Imaging and Image-Guided Therapy, Medical \\ University of Vienna, Vienna, Austria; ${ }^{3}$ Breast Imaging Service, Department of Radiology, Memorial Sloan Kettering Cancer \\ Center, New York, New York; ${ }^{4}$ Department of Surgery, Memorial Sloan Kettering Cancer Center, New York, New York; \\ ${ }^{5}$ Breast Medicine Service, Department of Medicine, Memorial Sloan Kettering Cancer Center, and Weill Cornell Medical College, \\ New York, New York; and ${ }^{6}$ Epidemiology and Biostatistics/Biostatistics, Memorial Sloan Kettering Cancer Center, New York, \\ New York
}

The PET Response Criteria in Solid Tumors (PERCIST) are not specific regarding the number of lesions that should be analyzed per patient. This study evaluated how the number of analyzed lesions affects response assessment in metastatic breast cancer. Methods: In 60 patients, response was assessed by the change in $S_{U} V_{\text {peak }}$, normalized to lean body mass, of the most ${ }^{18} \mathrm{~F}-\mathrm{FDG}$-avid lesion (PERCIST 1 ) and by the change in the sum of normalized SUV $V_{\text {peak }}$ for up to 5 lesions (PERCIST 5). The correlation between response by PERCIST and progression-free and disease-specific survival was evaluated. Results: In responders and nonresponders, the respective progression-free survival at 2 y was $37.26 \%$ and $6.43 \%$ for PERCIST $1(P<0.0001)$ and $33.65 \%$ and $7.14 \%$ for PERCIST $5(P<0.0001)$ and the respective disease-specific survival at 4 y was $58.96 \%$ and $25.44 \%$ for PERCIST $1(P<0.012)$ and $59.12 \%$ vs $20.01 \%$ for PERCIST $5(P<0.002)$. Conclusion: The number of analyzed lesions does not appear to have a major impact on the prognostic value of response assessment with ${ }^{18} \mathrm{~F}-\mathrm{FDG}$ PET/CT in metastatic breast cancer.

Key Words: breast cancer; ${ }^{18} \mathrm{~F}-\mathrm{FDG}$; PET; PERCIST; treatment response; prediction

J Nucl Med 2016; 57:1102-1104

DOI: 10.2967/jnumed.115.166629

$\mathbf{T}$ he use of ${ }^{18} \mathrm{~F}-\mathrm{FDG}$ PET/CT has shown significant promise for the restaging and therapy monitoring of many cancers, including metastatic (stage IV) breast cancer (1-4). However, standardization of response assessment by ${ }^{18} \mathrm{~F}$-FDG PET/CT is still lacking between the various studies. The PET Response Criteria in Solid Tumors (PERCIST) have defined a framework for the ${ }^{18} \mathrm{~F}-\mathrm{FDG}$ PET/CT evaluation of tumor response to therapy (5). PERCIST recommends

Received Nov. 30, 2015; revision accepted Feb. 17, 2016.

For correspondence or reprints contact: Wolfgang A. Weber, Memorial Sloan Kettering Cancer Center, 1275 York Ave., Box 77, New York, NY 10065.

E-mail: weberw@mskcc.org

Published online Mar. 16, 2016.

COPYRIGHT (C 2016 by the Society of Nuclear Medicine and Molecular Imaging, Inc. quantifying ${ }^{18} \mathrm{~F}$-FDG uptake based on SUV normalized to lean body mass (SUL) and measuring changes in the peak ${ }^{18}$ F-FDG uptake of lesions $\left(\mathrm{SUL}_{\text {peak }}\right)$. The preferred approach is to measure $\mathrm{SUL}_{\text {peak }}$ for the most ${ }^{18} \mathrm{~F}$-FDG-avid lesion on the baseline and follow-up studies and determine the relative change. Analysis of the sum of SUL peak for up to 5 lesions is suggested as an alternative. However, it remains unclear whether these two approaches to response assessment lead to a substantial difference in the clinical outcome predicted. Therefore, the aim of this study was to compare analysis of 1 lesion (PERCIST 1) versus analysis of up to 5 lesions (PERCIST 5) for predicting the outcome of stage IV breast cancer patients who undergo systemic therapy.

\section{MATERIALS AND METHODS}

The institutional review board approved and waived the informedconsent requirement for this retrospective single-institution study, which was compliant with the Health Insurance Portability Account Act.

\section{Patients}

The hospital information system of the Memorial Sloan Kettering Cancer Center was screened for patients who had stage IV breast cancer during 2007-2013 and fulfilled the following inclusion criteria: baseline ${ }^{18} \mathrm{~F}$-FDG PET/CT $28 \mathrm{~d}$ before initiation of therapy and follow-up and no more than 3 mo (mean, $2.4 \mathrm{mo}$ ) after initiation of therapy; either first- or second-line cytotoxic, targeted, or immunotherapeutic systemic therapy in a clinical trial or any hormonal therapy before cytotoxic, targeted, or immunotherapeutic systemic therapy; and measurable disease at baseline as defined by PERCIST (5). Patients were excluded if metastatic disease was limited to the brain or if no lesion on ${ }^{18} \mathrm{~F}$-FDG PET/CT exceeded the limits for minimum SUL as defined by PERCIST $(5)(1.5 \times$ liver SUL +2 SDs of liver SUL $)$ and uptake time differed by more than $30 \mathrm{~min}$ between the baseline and follow-up scans.

Sixty eligible patients (mean age, 53.4 y; range, 29-85 y) were identified. The electronic medical records in the hospital information system were reviewed, and the following characteristics were recorded for each patient: age at therapy, type of therapy, start date of systemic therapy, histologic type, tumor grade, receptor status, date of progression, and date of death or last follow-up. If death was caused by breast cancer, this fact was also recorded (Supplemental Table 1, available at http://jnm.snmjournals.org). 


\section{Imaging}

Before receiving the ${ }^{18} \mathrm{~F}-\mathrm{FDG}$ injection for PET/CT, the patients fasted for at least $6 \mathrm{~h}$. Patients whose plasma glucose level was less than $200 \mathrm{mg} / \mathrm{dL}$ were intravenously injected with $444-555 \mathrm{MBq}$ of ${ }^{18} \mathrm{~F}-\mathrm{FDG}$, and scanning began after an uptake period of $60-90 \mathrm{~min}$. One patient had a plasma glucose level higher than $200 \mathrm{mg} / \mathrm{dL}$ at baseline and at follow-up and was injected with $4 \mathrm{IU}$ of a short-acting insulin (Novolog; Novo Nordisk) before receiving the ${ }^{18}$ F-FDG injection (Supplemental Fig. 1). Details about the PET/CT scanners and imaging technique are provided in Supplemental Table 2.

\section{Image Analysis}

One experienced physician who was board-certified in radiology and nuclear medicine reviewed the ${ }^{18} \mathrm{~F}-\mathrm{FDG}$ PET/CT data. The reviewer was aware of the clinical diagnosis but not the clinical followup information. ${ }^{18} \mathrm{~F}-\mathrm{FDG}$ uptake was quantified by $\mathrm{SUL}_{\text {peak }}$, using PET-VCAR software suite 2.2 (Advantage Workstation; GE Healthcare). To determine SUL, the reviewer placed a sphere around a possible target lesion. Within this sphere, the software searches for the $1.0-\mathrm{cm}^{3}$ sphere that encompasses the voxels with the highest average SUL. This SUL is reported as $\mathrm{SUV}_{\text {peak. }}$ Liver SUL was measured in a 3-cm region of interest (5).

Response to therapy was classified as either complete metabolic response, partial metabolic response, stable metabolic disease, or progressive metabolic disease according to PERCIST. For the PERCIST 1 analysis, the lesion with the highest $\mathrm{SUL}_{\text {peak }}$ was identified on the baseline image and on the follow-up scan (not necessarily the same lesion). The percentage change in $\mathrm{SUL}_{\text {peak }}$ between the baseline and follow-up scans was used to determine response as defined by PERCIST. Briefly, an increase by $30 \%$ or more, a new metabolically active lesion, or unequivocal progression of a nontarget lesion $(\geq 30 \%$ increase in any lesion) was defined as progressive disease. A decrease by $30 \%$ or more was defined as partial response. A decrease to blood-pool level or less was defined as a complete response. When none of these criteria were met, the response was defined as stable disease. For the PERCIST 5 analysis, up to 5 target lesions (maximum of two per organ) were identified. Their sums on the baseline scan and on the follow-up scan were calculated, and the percentage change used to determine response as defined by PERCIST.

The patients underwent clinical follow-up and contrast-enhanced CT or PET/CT every 3 mo until progression, followed by routine follow-up until death. These data were used to assess progression-free survival (PFS) and disease-specific survival (DSS).

\section{Statistical Analysis}

Concordance between response assessments by PERCIST 1 and PERCIST 5 was evaluated using $\kappa$-statistics. For survival analysis, the data were dichotomized into responders (complete or partial response) and nonresponders (stable or progressive disease). Kaplan-Meier analysis was used to determine whether there was an association between treatment response and either PFS or DSS. The log-rank (Mantel-Cox) test was used to evaluate differences between Kaplan-Meier curves. Statistical analyses were performed using Prism 6 software (GraphPad). $P$ values of 0.05 or less were considered significant.

\section{RESULTS}

The response classification according to PERCIST 1 and PERCIST 5 is summarized in Table 1. For PERCIST 1, 28 patients had the same target lesion on both the baseline and the follow-up scans and 32 patients had a different target lesion. Response was discordant between PERCIST 1 and PERCIST 5 in only 3 patients $(5 \%$, weighted $\kappa=0.96$ ). These 3 patients were classified as having stable disease by PERCIST 1 but a partial response by PERCIST 5. One of the three died after 31 mo whereas the other two were still alive at the end of the follow-up period, with survival of 35 and 38 mo. Progressive disease was concordantly diagnosed by PERCIST 1 and PERCIST 5 in 13 patients. The reasons for progressive disease were new lesions in 2 cases, unequivocal progression of nontarget lesions

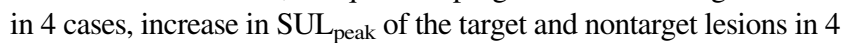
cases, and increase in SUL peak of the target lesions in 3 cases.

Response according to both PERCIST 1 and PERCIST 5 found highly significant differences in both 2-y PFS and 4-y DSS between responders and nonresponders (Figs. 1 and 2). The median follow-up time for all patients was 25.0 mo (range, 4.0-52 mo). Forty-nine patients $(81 \%)$ progressed during the follow-up period, after a median interval of 8.3 mo (range, 01.3-39.7 mo). Thirty patients $(50 \%)$ died of breast cancer during the follow-up period, at a median interval of $17 \mathrm{mo}$ (range, 4-36 mo).

The respective 2-y PFS for responders and nonresponders was $37.26 \%$ and $6.43 \%$ for PERCIST $1(P<0.0001)$ and $33.65 \%$ and $7.14 \%$ for PERCIST $5(P<0.0001)$ (Fig. 1$)$. The median time to progression for nonresponders was 3.03 mo for PERCIST 1 and 2.8 mo for PERCIST 5. The median time to progression for responders was 16.1 mo for PERCIST 1 and 14.3 mo for PERCIST 5. The respective 4-y DSS for responders and nonresponders was $58.96 \%$ and $25.44 \%$ for PERCIST $1(P<0.008)$ and $59.12 \%$ and $20.01 \%$ for PERCIST 5 ( $P<0.0009$ ) (Fig. 2). The median DSS for nonresponders was 21 mo both for PERCIST 1 and for PERCIST 5 and had not been reached by the end of the follow-up for responders. The 2-y PFS for responders $(P=0.832)$ and nonresponders $(P=$ $0.667)$ did not significantly differ between PERCIST 1 and PERCIST 5 , nor did the 4-y DSS for responders $(P=0.948)$ or nonresponders $(P=0.604)$.

TABLE 1

Correlation Between Response Assessment by PERCIST 1 and PERCIST 5

\begin{tabular}{lccccc}
\hline & & \multicolumn{2}{c}{ PERCIST 1 } \\
\cline { 2 - 6 } \multicolumn{1}{c}{ PERCIST 5 } & Complete response & Partial response & Stable disease & Progressive disease & Sum \\
\hline Complete response & 19 & 0 & 0 & 0 & 19 \\
\hline Partial response & 0 & 10 & 3 & 0 & 13 \\
Stable disease & 0 & 0 & 15 & 0 & 13 \\
Progressive disease & 0 & 0 & 18 & 13 & 60 \\
Sum & 19 & 10 & & 13 \\
\hline
\end{tabular}




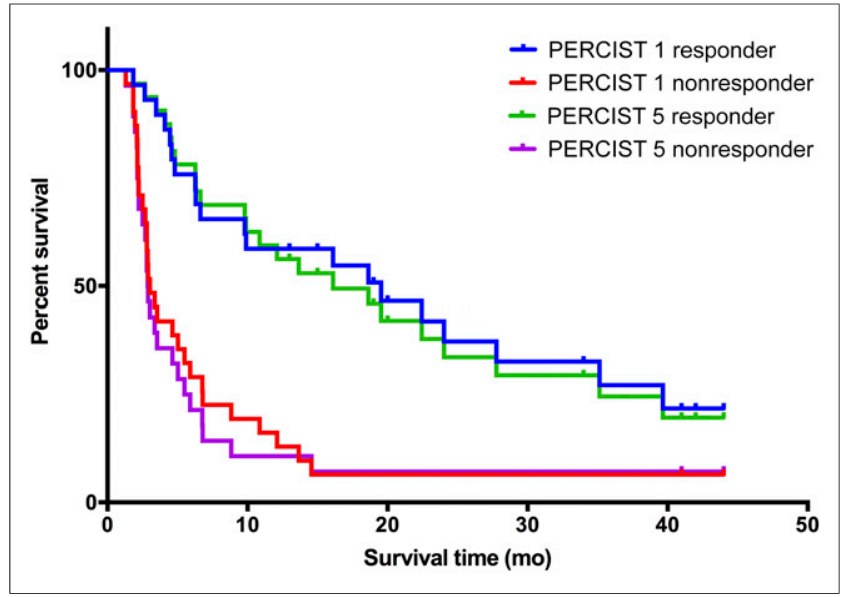

FIGURE 1. PFS by tumor response.

In 16 patients, the percentage difference in normal-liver SUL was greater than recommended by PERCIST (5). Excluding these patients had no impact on the results (Supplemental Figs. 2 and 3).

\section{DISCUSSION}

The use of ${ }^{18}$ F-FDG PET and PET/CT for response assessment in breast cancer has been extensively studied. The initial studies focused on patients with locally advanced breast cancer who underwent neoadjuvant therapy (6-8). In that setting, the question of the number of lesions to analyze is not particularly relevant, as there is generally a single dominant breast mass and the histopathology of this mass is the reference standard for response assessment on ${ }^{18} \mathrm{~F}$-FDG PET/CT. More recently, ${ }^{18} \mathrm{~F}-\mathrm{FDG} \mathrm{PET} / \mathrm{CT}$ has also shown promising results in metastatic breast cancer $(2-4,9-11)$. In this setting, patients may show so many lesions on ${ }^{18} \mathrm{~F}-\mathrm{FDG}$ PET/CT that it becomes quite cumbersome to measure ${ }^{18} \mathrm{~F}-\mathrm{FDG}$ uptake for each of them. However, to our knowledge the number of lesions that needs to be measured to accurately assess response has not yet been determined. Therefore, PERCIST, although recommending an analysis of the most metabolically active lesion, also encourages averaging SUL for up to 5 lesions (5).

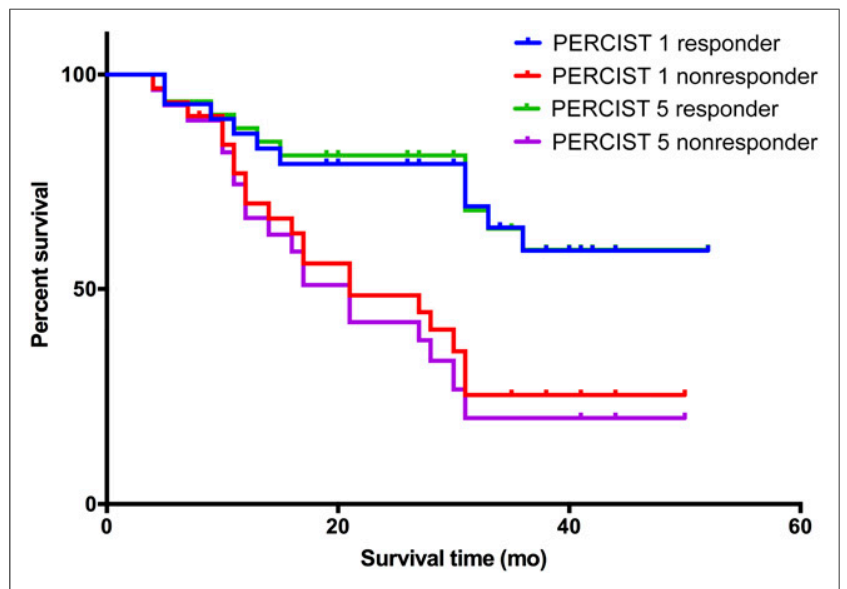

FIGURE 2. DSS by tumor response.
The present study indicated that the only effect of analyzing 1 rather than 5 lesions is a minimal decrease in the frequency of partial response. Fewer patients with partial response are expected for PERCIST 1, because it chooses the lesion with the highest $\mathrm{SUL}_{\text {peak }}$ on the follow-up scan for calculating the percentage change in ${ }^{18} \mathrm{~F}$-FDG uptake. This change will tend to be smaller than the change in SUL summed for several lesions, as used by PERCIST 5. For similar reasons, one might expect a higher frequency of progressive disease for PERCIST 1 than for PERCIST 5. However, the fact that progressive disease in the present study was usually attributable to the appearance of new lesions or the progression of nontarget lesions explains why there was no difference between PERCIST 1 and PERCIST 5 for this category.

\section{CONCLUSION}

In metastatic breast cancer, ${ }^{18} \mathrm{~F}-\mathrm{FDG} \mathrm{PET} / \mathrm{CT}$ is a robust method for monitoring the response to therapy. In the current study, the exact number of analyzed lesions did not have a major influence on the prognostic value of response by ${ }^{18} \mathrm{~F}-\mathrm{FDG}$ PET/ CT. Larger prospective trials will be helpful in validating these results.

\section{DISCLOSURE}

The costs of publication of this article were defrayed in part by the payment of page charges. Therefore, and solely to indicate this fact, this article is hereby marked "advertisement" in accordance with 18 USC section 1734 . No potential conflict of interest relevant to this article was reported.

\section{REFERENCES}

1. Weber WA. Assessing tumor response to therapy. J Nucl Med. 2009;50(suppl 1):1S-10S.

2. Schwarz JD, Bader M, Jenicke L, Hemminger G, Janicke F, Avril N. Early prediction of response to chemotherapy in metastatic breast cancer using sequential ${ }^{18}$ F-FDG PET. J Nucl Med. 2005;46:1144-1150.

3. Groheux D, Giacchetti S, Delord M, et al. Prognostic impact of ${ }^{18}$ F-FDG $\mathrm{PET} / \mathrm{CT}$ staging and of pathological response to neoadjuvant chemotherapy in triple-negative breast cancer. Eur J Nucl Med Mol Imaging. 2015;42: $377-385$.

4. Mortazavi-Jehanno N, Giraudet AL, Champion L, et al. Assessment of response to endocrine therapy using FDG PET/CT in metastatic breast cancer: a pilot study. Eur J Nucl Med Mol Imaging. 2012;39:450-460.

5. Wahl RL, Jacene H, Kasamon Y, Lodge MA. From RECIST to PERCIST: evolving considerations for PET response criteria in solid tumors. J Nucl Med. 2009;50(suppl 1):122S-150S.

6. Smith IC, Welch AE, Hutcheon AW, et al. Positron emission tomography using $\left[{ }^{18} \mathrm{~F}\right]$-fluorodeoxy-D-glucose to predict the pathologic response of breast cancer to primary chemotherapy. J Clin Oncol. 2000;18:1676-1688.

7. Schelling M, Avril N, Nahrig J, et al. Positron emission tomography using $\left[{ }^{18} \mathrm{~F}\right]$ fluorodeoxyglucose for monitoring primary chemotherapy in breast cancer. $J$ Clin Oncol. 2000;18:1689-1695.

8. Mankoff DA, Dunnwald LK, Gralow JR, et al. Changes in blood flow and metabolism in locally advanced breast cancer treated with neoadjuvant chemotherapy. J Nucl Med. 2003;44:1806-1814.

9. Cachin F, Prince HM, Hogg A, Ware RE, Hicks RJ. Powerful prognostic stratification by $\left[{ }^{18} \mathrm{~F}\right]$ fluorodeoxyglucose positron emission tomography in patients with metastatic breast cancer treated with high-dose chemotherapy. J Clin Oncol. 2006;24:3026-3031.

10. Ulaner GA, Eaton A, Morris PG, et al. Prognostic value of quantitative fluorodeoxyglucose measurements in newly diagnosed metastatic breast cancer. Cancer Med. 2013;2:725-733.

11. Morris PG, Ulaner GA, Eaton A, et al. Standardized uptake value by positron emission tomography/computed tomography as a prognostic variable in metastatic breast cancer. Cancer. 2012;118:5454-5462. 